\title{
Inducción de trabajo de parto con cervix desfavorable Experimento clínico aleatorizado doble ciego Oxitocina vs Misoprostol
}

\author{
Javier Ardila Montealegre; Luis Fernando Botero; Germán Sabogal*
}

\begin{abstract}
RESUMEN
Se compara la eficacia y seguridad del Misoprostol con la Oxitocina para inducción de pacientes con HIE, RPM y Embarazo Prolongado y cérvix desfavorable (Bishop menor o igual a 6), que tuvieran una edad entre 15 y 45 años; mediante un experimento clínico aleatorizado doble-ciego con doble enmascaramiento. Se reclutan 182 pacientes que se asignan aleatoriamente a los grupos una vez fueran elegibles y de acuerdo a los criterios de inclusión y exclusión (Antecedente de cesárea y/o cirugía previa en el cuerpo uterino o en el cérvix, sufrimiento fetal agudo o crónico, placenta previa, corioamnionitis, distocias, embarazo múltiple). Se aplicaron en el análisis el T-Test, Pearson Chi2 según el tipo de variable que se analizaba, encontrando que el Misoprostol mostró ser más eficaz para inducir pacientes con cérvix desfavorable, se disminuyó la incidencia de cesáreas por inducción fallida y no se demostró un aumento en la incidencia de SFA en el grupo del misoprostol.
\end{abstract}

\section{PALABRAS CLAVES: Misoprostol, oxitocina, inducción trabajo de parto, cervix desfavorable}

\section{SUMMARY}

It is compared the effectiveness and security of the Misoprostol with the Oxitocina for labor induction of patient with Hypertension Induced pregnancy to term, Premature Rupture Membranes to term and Prolonged pregnancy with unfavorable cérvix $(B i s h o p<o ́$ $=6$ ), age between 15 and 45 years old, normal fetal heart rate tracings by means of a double-blind randomized clinical trial. 182 patients are recruited that are assigned randomly once to the groups they were eligible and according to the inclusion approaches and exclusion (Antecedent of Caesarean y/o previous surgery in the uterine body or in the cérvix, unnormal fetal heart rate tracings, previous placenta, corioamnionitis, distocias, multiple pregnancy). They were applied in the analysis the T-Test, Pearson Chi2 according to the variable type that was analyzed, finding that the Misoprostol showed to be more effective to induce patient with unfavorable cérvix, Diminished the incidence of Caesarean for bankrupt induction and increase was not demonstrated in the incidence of fetus affected with hipoxy in the group of the misoprostol.

KEY WORDS: Misoprostol, oxytocina, labor induction, cervix unfavorable.

En la obstetricia actual, es cada vez más frecuente el hecho de tenernos que enfrentar a situaciones que requieren la inducción del trabajo de parto como parte del manejo de la paciente y su feto. Tales son los casos de la hipertensión inducida por el embarazo, la ruptura prematura de membranas y el embarazo prolongado. Sin embargo, estas decisiones clínicas de desembarazar a una paciente mediante la inducción de las contracciones tiene un factor de considerable importancia como son las características del cuello uterino en el momento de tomar la determinación, puesto que dependiendo de estas, se pronostica el éxito con la terminación de todo el proceso con un parto vaginal; o por el contrario su fracaso con una cesárea que generalmente se indica por una inducción fallida, entendi-

Universidad Javeriana - Hospital San Ignacio. Departamento de Obstetricia y Genecología. da por ausencia de contracciones después de llegar a ciertos límites de tiempo o de dosis del medicamento útero-tónico; o ausencia de cambios en el cuello uterino (Borramiento y dilatación) a pesar de generar dinámica uterina con el medicamento administrado.

Una de las posibles explicaciones para las inducciones fallidas en cérvix desfavorable es el uso de la tradicional oxitocina que como todos sabemos ha mostrado no ser tan efectiva en estos casos como las prostaglandinas de uso más reciente en nuestra área; y específicamente el Misoprostol el cual ha venido siendo utilizado de manera empírica y casi subrepticia en nuestro medio. Teniendo en cuenta esto y además la evidencia en la literatura mundial que muestra gran cantidad de grupos utilizando este medicamento para inducción de trabajo de parto, hemos querido realizar un trabajo original que aclare las inquietudes respectivas al tema y desde luego permita tomar una decisión más racional acerca de su uso con sus respectivas ventajas y desventajas. 


\section{Objetivos}

1. Evaluar la efectividad y seguridad del Misoprostol en inducción de trabajo de parto.

2. Disminuir la incidencia de cesáreas en inducción de trabajo de parto con cérvix desfavorable.

3. Describir los efectos secundarios del Misoprostol en la inducción del trabajo de parto.

\section{Diseño de Investigación}

Se realizó un experimento clínico aleatorizado dobleciego con doble enmascaramiento que comparó pacientes con cérvix desfavorable sometidas a inducción del trabajo de parto con Oxitocina y con Misoprostol.

El protocolo tuvo tres fases en su desarrollo, a saber:

1. Estandarización, para establecer mecánicas de desempeño iguales en los conceptos entre las diferente personas involucradas en el desarrollo del protocolo. Esta etapa del protocolo puntualizó temas como valoración del índice de Bishop, aclarar criterios diagnósticos en las patologías incluidas, manejo y seguimiento de las pacientes con respecto al esquema de inducción utilizado y definición clara y concisa de conceptos tales como inducción fallida, desproporción céfalo-pélvica, Sufrimiento fetal agudo, polisistolia etc.

2. Estudio piloto, con el ánimo de identificar dificultades técnicas que pudieran hacer perder validez a los resultados.

3. Medidas correctivas de las dificultades previamente identificadas, como por ejemplo nombrar la persona encargada de hacer los paquetes de inducción (Oxitocina + placebo y Misoprostol + placebo), hacer el seguimiento estricto del consecutivo correspondiente a la tabla de números aleatorios y desde luego la recolección de la información para registrarla en las bases de datos.

\section{Criterios de inclusión}

1. Edad entre 15 y 45 años

2. Indicación de inducción dada por: Ruptura Prematura de Membranas Hipertensión inducida por el embarazo Embarazo Prolongado

3. Indice de Bishop menor o igual que 6

4. Consentimiento informado de la paciente

\section{Criterios de exclusión}

1. Antecedente de cesárea

2. Corioamnionitis

3. Distocia de presentación

4. Antecedente de cirugía de cérvix

5. Sufrimiento fetal agudo o crónico

6. Antecedente de miomectomía

7. Placenta previa

8. Cirugía correctiva del piso pélvico

9. Situaciones médicas y/o quirúrgicas que contraindiquen el trabajo de parto

1.0. Carcinoma de cérvix

11. Embarazo múltiple
12. Gran multiparidad (Mayor de 5 gestaciones)

13. No aceptación de la paciente

\section{Tamaño de Muestra}

Se calcula el tamaño de muestra teniendo en cuenta una diferencia del $25 \%$ en la incidencia de cesáreas entre los grupos propuestos, un poder del $80 \%$, un nivel alfa de 0,05 y dos colas.

\section{Materiales y Métodos}

El protocolo se desarrolla en el Hospital Universitario de San Ignacio con las pacientes que ingresaban a sala de partos, siguiendo el siguiente esquema:

Una vez la paciente calificara como elegible de acuerdo a los criterios anteriormente expuestos y aceptara estar ser incluida en el estudio se le realizaba comprobación del bienestar fetal mediante una prueba sin estrés que fuera reactiva. Una vez se comprobaba ausencia de compromiso fetal se asignó de manera aleatoria a uno de los dos grupos de inducción (Oxitocina o Misoprostol).

A toda paciente asignada a la inducción se le administraba un "paquete de inducción" previamente hecho que constaba de:

Lactato Ringer 500 c.c con 5 UI de Oxitocina diluidas y una pequeña bolsa no transparente con un placebo (iguales características físicas que media tableta de Misoprostol), en caso que le correspondiera la inducción con la Oxitocina; ó Lactato Ringer 500 c.c con 2 c.c de agua destilada diluida y una pequeña bolsa no transparente con media tabaleta de Misoprostol (100 microgramos). En ambas situaciones se colocó a la paciente la media tableta en el fondo de saco posterior por el médico interno de turno (que no tendría que ver con el manejo y seguimiento de la paciente) y un equipo de bomba de infusión iniciando a $2 \mathrm{mUL} / \mathrm{min}$ y aumentando otro tanto cada 20 minutos hasta una dosis máxima de $36 \mathrm{mUI} / \mathrm{min}$ o hasta que completara 8 horas sin dinámica uterina.

Las personas encargadas del manejo directo de la paciente fueron el médico residente y el especialista, los cuales no sabían cual era el principio activo responsable de la inducción.

Las pacientes reclutadas en el protocolo tuvieron nueva monitoría fetal una vez iniciaron actividad uterina.

El análisis estadístico se efectuó con Stata practicando T-Test y Pearson Chi2 según el tipo de variables que se analizaran.

\section{Resultados}

Se reclutaron 182 pacientes de las cuales las características generales están descritas en la tabla 1.

Los tipos de parto en cada grupo se distribuyeron como indica la tabla 2, hallando una diferencia estadísticamente significativa en los partos instrumentados, los cuales fueron mayores en el grupo del Misoprostol y en la cesárea, la cual fue considerablemente mayor en el grupo de la Oxitocina. (Tabla 2).

El tiempo desde el inicio de la actividad uterina hasta el parto fue $16 \%$ menos en el grupo del misoprostol, situación esta que podría ser ventajosa en aquellas pa- 
Tabla 1

CARACTERÍSTICAS DE LA POBLACIÓN ESTUDIO

\begin{tabular}{|c|c|c|}
\hline & MISOPROSTOL & OXITOCINA \\
\hline EDAD & $\begin{array}{cc}20-30 & (72,5 \%) \\
\mathbf{1 5}-\mathbf{1 9} & (\mathbf{9 , 9 \% )} \\
>30 & (16,5 \%)\end{array}$ & 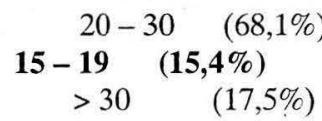 \\
\hline $\begin{array}{c}\text { EMBARAZO } \\
\text { PROLONGADO } \\
\text { RUTURA PREMATURA DE } \\
\text { MEMBRANAS } \\
\text { HIPERTENSION } \\
\text { INDUCIDA POR EL EMBARAZO }\end{array}$ & $\begin{array}{ll}60 & (50 \%) \\
07 & (46,7 \%) \\
24 & (51,1 \%)\end{array}$ & $\begin{array}{ll}60 & (50 \%) \\
08 & (53,3 \%) \\
23 & (48,9 \%)\end{array}$ \\
\hline
\end{tabular}

cientes que requieren de un trabajo de parto más corto que el usual como por ejemplo las que padecen hipertensión inducida por el embarazo. (Tabla 3).

Tabla 2

TIPOS DE PARTO

\begin{tabular}{|lccc|}
\hline & EUTOCICO INSTRUMENTADO & CESAREA \\
\hline OXITOCINA & 29 & $\mathbf{1 6}$ & $\mathbf{4 6}$ \\
MISOPROSTOL & 47 & 26 & $\mathbf{1 8}$ \\
\hline
\end{tabular}

PEARSON CHI2 $=18,8941 \quad \mathrm{P}=0,0001(\mathrm{~S})$

Tabla 3

TIEMPO DESDE EL INICIO DEL TRABAJO DE PARTO HASTA EL PARTO

\begin{tabular}{|cc|}
\hline & MEDIA (HORAS) \\
\hline OXITOCINA & 10,1 \\
MISOPROSTOL & 8,5 \\
\hline
\end{tabular}

$$
\text { T-TEST }=3,4723 \quad P=0,0006(S) .
$$

El grupo de inducción con oxitocina presentó una mayor incidencia de cesáreas siendo significativa desde el punto de vista tanto estadístico como clínico en aquellas indicadas por inducción fallida y desproporción céfalo-pélvica. (Tabla 4).

El Sufrimiento fetal agudo se presentó en los dos grupos por igual sin lograr hallar una diferencia estadísticamente significativa. (Tabla 5)

Tabla 4

INDICACIONES DE CESÁREA

\begin{tabular}{|lccc|}
\hline & INDUCC. & & \\
& FALLIDA & S.F.A & D.C.P \\
\hline OXITOCINA & 25 & 03 & $\boldsymbol{1 8}$ \\
MISOPROSTOL & 07 & 04 & $\mathbf{0 8}$ \\
\hline
\end{tabular}

PEARSON CHI2= 18,8941
Tabla 5

DISTRIBUCIÓN DEL SUFRIMIENTO FETAL AGUDO

\begin{tabular}{|lcc|}
\hline & POSITIVO & NEGATIVO \\
\hline OXITOCINA & 03 & 88 \\
MISOPROSTOL & 04 & 87 \\
\hline
\end{tabular}

PEARSON CHI $2=0,1486 \quad P<0,7(N S)$

Un evidente hallazgo en la inducción con Misoprostol es la polisistolia cuando se compara con la Oxitocina, (Tabla 6), sin embargo, la relación de la polisistolia con la aparición de Sufrimiento Fetal Agudo no es significativa, lo cual hace pensar en que esta no reviste importancia clínica (Tabla 7).

Tabla 6

DISTRIBUCIÓN DE LA POLISISTOLIA

\begin{tabular}{|lcc|}
\hline & PRESENTE & AUSENTE \\
\hline OXITOCINA & 08 & 83 \\
MISOPROSTOL & $\mathbf{2 1}$ & 70 \\
\hline
\end{tabular}

PEARSON CHI2=6,7696 $\quad \boldsymbol{P}<0,0097(\boldsymbol{S})$

Tabla 7

RELACIÓN DE LA POLISISTOLIA CON EL SUFRIMIENTO FETAL AGUDO

\begin{tabular}{|lcc|}
\hline & S.F.A & NO S.F.A \\
\hline POLISISTOLIA & 02 & 27 \\
NO POLISISTOLIA & 05 & 147 \\
\hline
\end{tabular}

PEARSON CHI2 $=0,8523$

$P<0,356(N S)$ 
Tabla 8

TIPOS DE PARTO EN HIPERTENSIÓN INDUCIDA POR EL EMBARAZO

\begin{tabular}{|lccc|}
\hline & \multicolumn{4}{c|}{ EUTOCICO INSTRUMENTADO } & CESAREA \\
\hline OXITOCINA & 05 & 07 & $\boldsymbol{1 1}$ \\
MISOPROSTOL & 11 & 13 & $\boldsymbol{0 0}$ \\
\hline
\end{tabular}

PEARSON CHI $2=15,0355 \quad \boldsymbol{P}=\mathbf{0 , 0 0 1}(\mathrm{S})$

Los tipos de parto en la diferentes patologías nos muestra una diferencia significativa especialmente en la Hipertensión inducida por el embarazo y el Embarazo Prolongado, donde la cesárea es más frecuente en el grupo de la Oxitocina (Tablas 8 y 10). Con respecto al grupo de la Ruptura Prematura de Membranas, es posible que no se haya encontrado ninguna diferencia por la escasa cantidad de pacientes en este grupo (tabla 9)

Tabla 9

TIPOS DE PARTO EN RUPTURA PREMATURA DE MEMBRANAS

\begin{tabular}{|lccc|}
\hline & EUTOCICO INSTRUMENTADO & CESAREA \\
\hline OXITOCINA & 04 & 01 & 03 \\
MISOPROSTOL & 01 & 03 & 03 \\
\hline
\end{tabular}

PEARSON CHI2= 2,7455

$P<0,253(N S)$
Tabla 10

TIPOS DE PARTO EN EMBARAZO PROLONGADO

\begin{tabular}{|lccc|}
\hline & \multicolumn{2}{c}{ EUTOCICO INSTRUMENTADO } & CESAREA \\
\hline OXITOCINA & 20 & 08 & $\mathbf{3 2}$ \\
MISOPROSTOL & 35 & 10 & $\mathbf{1 5}$ \\
\hline
\end{tabular}

PEARSON CHI2 $=10,4621 \quad P=0,005(S)$

\section{Conclusiones}

1. El Misoprostol mostró ser más eficaz para inducir exitosamente pacientes con cérvix desfavorable.

2. El Misoprostol disminuye la incidencia de cesáreas por inducción fallida.

3. No se demostró mayor cantidad de casos de Sufrimiento Fetal Agudo en el grupo de pacientes que se indujeron con Misoprostol.

4. Se sugiere un mayor beneficio del Misoprostol especialmente cuando se utiliza en pacientes que padecen Hipertensión inducida por el embarazo y Embarazo Prolongado; no así en pacientes con Ruptura Prematura de Membranas.

5. El Misoprostol se constituye en una alternativa más eficaz que la Oxitocina para inducción de trabajo de parto con cérvix desfavorable y con igual seguridad que esta última.

6. Las pacientes que requieren de un trabajo de parto más corto que el normal pueden beneficiarse de la inducción con Misoprostol.

\section{BIBLIOGRAFIA}

1. El Refaey H; Jauniaux E. Methods of induction of labour. Curr Opin Obstet Gynecol, 1997; 9(6): 375-8.

2. Bauer TA; Brown DL; Chai LK . Vaginal misoprostol for term labor induction [see comments] Ann Pharmacother, 1997; 31(11): 1391-3.

3. Summers L. Methods of cervical ripening and labor induction. $\mathbf{J}$ Nurse Midwifery, 1997; 42(2): 71-85.

4. Sanchez Ramos L; Kaunitz AM; Wears RL; Delke I; Gaudier FL Misoprostol for cervical ripening and labor induction: a meta-analysis. Obstet Gynecol, 1997; 89(4): 633-42.

5. Sanchez Ramos L; Peterson DE; Delke I; Gaudier FL; Kaunitz AM Labor induction with prostaglandin E1 misoprostol compared with dinoprostone vaginal insert: a randomized trial. Obstet Gynecol, 1998; 91(3): 401-5.

6. Mundle WR; Young DC. Vaginal misoprostol for induction of labor: a randomized controlled trial. Obstet Gynecol, 1996; 88(4): 521-25.

7. Herabutya Y; O Prasertsawat P; Pokpirom J. A comparison of intravaginal misoprostol and intracervical prostaglandin E2 gel for ripening of unfavorable cervix and labor induction. J Obstet Gynaecol Res, 1997; 23(4): 369-74.

8. Kadanali S; Küçüközkan T; Zor N; Kumtepe Y. Comparison of labor induction with misoprostol vs. oxytocin/prostaglandin E2 in term pregnancy. Int J Gynaecol Obstet, 1996; 55(2): 99-104.

9. Magtibay PM; Ramin KD; Harris DY; Ramsey PS; Ogburn PL Jr. Misoprostol as a labor induction agent. J Matern Fetal Med, 1998; 7(1): 15-8.

10. Wing DA; Ortiz Omphroy G; Paul RH. A comparison of intermittent vaginal administration of misoprostol with continuous dinoprostone for cervical ripening and labor induction. Am J Obstet Gynecol, 1997; 177(3): 612-8.

11. Gottschall DS; Borgida AF; Mihalek JJ; Sauer F; Rodis JF. A randomized clinical trial comparing misoprostol with prostaglandin E2 gel for preinduction cervical ripening. Am J Obstet Gynecol, 1997; 177(5): 1067-70.

12. Ozgür K; Kizilates A; Uner M; Erman O; Trak B. Induction of labor with intravaginal misoprostol versus intracervical dinoprostone. Arch Gynecol Obstet, 1997; 261(1): 9-13.

13. Surbek DV; Boesiger H; Hoesli I; Pavic N; Holzgreve W. A doubleblind comparison of the safety and efficacy of intravaginal misoprostol and prostaglandin E2 to induce labor. Am J Obstet Gynecol, 1997; 177(5): 1018-23.

14. Windrim R; Bennett K; Mundle W; Young DC. Oral administration of misoprostol for labor induction: a randomized controlled trial [see comments] Obstet Gynecol, 1997; 89(3): 392-7.

15. Farah LA; Sanchez Ramos L; Rosa C; Del Valle GO; Gaudier FL; Delke I; Kaunitz AM. Randomized trial of two doses of the prostaglandin El analog misoprostol for labor induction. Am J Obstet Gynecol, 1997; 177(2): 364-9; discussion 369-71.

16. Toppozada MK; Anwar MY; Hassan HA; el Gazaerly WS. Oral or vaginal misoprostol for induction of labor. Int J Gynaecol Obstet, 1997; 56(2): 135-9

17. Escudero $\mathrm{F}$; Contreras $\mathrm{H}$ A comparative trial of labor induction with misoprostol versus oxytocin. Int J Gynaecol Obstet, 1997; 57(2): 139-43.

18. Wing DA; Paul RH. A comparison of differing dosing regimens of vaginally administered misoprostol for preinduction cervical ripening and labor induction [published erratum appears in Am $\mathbf{J}$ Obstet 
Gynecol 1997 Jun;176(6):1423]. Am J Obstet Gynecol, 1996; Jul, $175: 1,158-64$

19. Lee HY. A randomised double-blind study of vaginal misoprostol vs dinoprostone for cervical ripening and labour induction in prolonged pregnancy. Singapore Med J, 1997; 38(7): 292-4.

20. Sanchez Ramos L; Chen AH; Kaunitz AM; Gaudier FL; Delke I. Labor induction with intravaginal misoprostol in term premature rupture of membranes: a randomized study. Obstet Gynecol, 1997; 89(6): 909-12.

21. Wing DA; Lovett K; Paul RH. Disruption of prior uterine incision following misoprostol for labor induction in women with previous cesarean delivery. Obstet Gynecol, 1998; 91(5): 828-30.

22. Srisomboon J; Piyamongkol W; Aiewsakul P. Comparison of intracervical and intravaginal misoprostol for cervical ripening and labour induction in patients with an unfavourable cervix. J Med Assoc Thai, 1997; 80(3): 189-94.

23. Ngai SW; To WK; Lao T; Ho PC. Cervical priming with oral misoprostol in pre-labor rupture of membranes at term. Obstet Gynecol, 1996; 87(6): 923-6.
24. Carlan SJ; Bouldin S; OBrien WF. Extemporaneous preparation of misoprostol gel for cervical ripening: a randomized trial. Obstet Gynecol, 1997; 90(6): 911-5.

25. Srisomboon J; Tongsong T; Tosiri V. Preinduction cervical ripening with intravaginal prostaglandin E1 methyl analogue misoprostol: a randomized controlled trial. J Obstet Gynaecol Res, 1996; 22(2): 119-24.

26. Kramer RL; Gilson GJ; Morrison DS; Martin D; Gonzales JL; Qualls CR. A randomized trial of misoprostol and oxytocin for induction of labor: safety and efficacy [see comments]. Obstet Gynecol, 1997; 89(3): 387-91.

27. Buser D; Mora G; Arias F. A randomized comparison between misoprostol and dinoprostone for cervical ripening and laborinduction in patients with unfavorable cervices. Obstet Gynecol, 1997; 89(4): 581-5. 